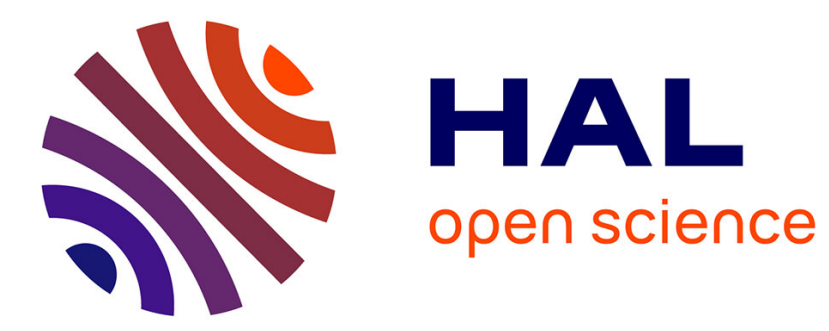

\title{
Simultaneous stabilization of single-input nonlinear systems with bounded controls
}

\author{
Abderrahman Iggidr, Mohamed Oumoun
}

\section{To cite this version:}

Abderrahman Iggidr, Mohamed Oumoun. Simultaneous stabilization of single-input nonlinear systems with bounded controls. International Journal of Dynamics and Control, 2021, 9, pp.550-556. 10.1007/s40435-020-00658-3 . hal-02943170

\section{HAL Id: hal-02943170 \\ https://hal.inria.fr/hal-02943170}

Submitted on 18 Sep 2020

HAL is a multi-disciplinary open access archive for the deposit and dissemination of scientific research documents, whether they are published or not. The documents may come from teaching and research institutions in France or abroad, or from public or private research centers.
L'archive ouverte pluridisciplinaire HAL, est destinée au dépôt et à la diffusion de documents scientifiques de niveau recherche, publiés ou non, émanant des établissements d'enseignement et de recherche français ou étrangers, des laboratoires publics ou privés. 


\title{
Simultaneous Stabilization of Single-Input Nonlinear Systems With Bounded Controls
}

\author{
Abderrahman Iggidr*1 and Mohamed Oumoun ${ }^{\dagger 2}$ \\ ${ }^{1}$ Université de Lorraine, CNRS, Inria, IECL, F57000 Metz, \\ France \\ ${ }^{2}$ Cadi Ayyad University, National School of Applied Sciences, \\ Marrakech, Morocco
}

Received: 6 December 2019 / Accepted: 9 June 2020

The final publication is available at link.springer.com: https://link.springer.com/article/10.1007/ s40435-020-00658-3

\begin{abstract}
In this paper, the simultaneous stabilization of single-input nonlinear systems with bounded controls is considered. Using the Lyapunov approach and based on Lin-Sontag's formula for bounded and continuous stabilizers for affine nonlinear systems, a constructive universal formula for the bounded simultaneous stabilization of single-input nonlinear systems is presented explicitly. An illustrative example is given to demonstrate the validity of the method.
\end{abstract}

Keywords. Simultaneous stabilization, nonlinear systems, control Lyapunov function, bounded state feedback.

\footnotetext{
*Abderrahman.Iggidr@inria.fr

${ }^{\dagger}$ Corresponding author: m.oumoun@uca.ac.ma
} 


\section{Introduction}

Stabilization of control systems is one of the most important topics in control theory. The most useful tool in stability analysis and stabilizing control design is the Lyapunov approach. In practice, this approach consists in finding a feedback law together with a positive definite function which decreases along the trajectories of the closed-loop system. Since it is not always easy to find such a function, the stabilizability of nonlinear systems has been widely studied in the past decades by many authors by using the so-called control Lyapunov function (CLF) ( see $[7,10,11,20,15,16]$ and the references therein).

The CLF was introduced by Artstein [1] and made a tremendous impact on stabilization theory. Historically, one of the most significant results using the technique of CLF was the "universal" formula given by Sontag in [14] and revisited later on by Lin and Sontag [6].

Among the various types of problem treated in the stabilization, the simultaneous stabilization problem which is concerned with the design of a single controller to simultaneously stabilize a family of systems has received a good deal of attention. For linear simultaneous stabilization problems, many interesting results have been presented in the literature (see $[2,3,5,8,9,12,13]$ )

For nonlinear systems, the simultaneous stabilization problem is more difficult to solve. Since most practical systems exhibit nonlinear behaviors, it is important to study the nonlinear case. To our knowledge, there are not so many papers on the subject. We can cite Ho-Mock-Qai and Dayawansa [4] who showed the existence of simultaneous stabilization (non-asymptotically) controllers for a set of nonlinear systems. The simultaneous stabilization problem was investigated for a set of nonlinear port-controlled Hamiltonian systems in [17]. Wu in [19] designed a simultaneously quadratically stabilizing state feedback controllers for a collection of single-input discrete-time nonlinear systems. Based on Sontag's formula for continuous stabilizers [14], Wu [18] presented a necessary and sufficient condition for the existence of simultaneously stabilizing controllers of single-input nonlinear systems. Under a sufficient but not necessary condition, Zhang et al. [21] presented a new simultaneously stabilizing state feedback controller.

Motivated by the works [18] and [21], this paper considers the simultaneous stabilization of single-input nonlinear systems with controls taking values from a bounded interval. Under the same necessary and sufficient condition stated in [18], and based on Lin-Sontag's formula for bounded and continuous stabilizers [6], we present a simple proof and a simultaneously stabilizing bounded feedback for single-input nonlinear systems. The stabilizing feedbacks are given explicitly. 


\section{Problem formulation and preliminaries}

Let us consider the following collection of affine systems described by

$$
S_{i}: \dot{x}=f_{i}(x)+u g_{i}(x), \quad i \in \mathcal{I}=\{1,2, \ldots, m\},
$$

where $x \in \mathbb{R}^{n}$ is the state, $f_{i}($.$) and g_{i}($.$) are smooth functions with f_{i}(0)=0$. Controls take values in the open interval

$$
\mathcal{B}_{1}=\{u \in \mathbb{R} \mid-1<u<1\} .
$$

Our objective is to find a single feedback law $p: \mathbb{R}^{n} \rightarrow \mathcal{B}_{1}$ with the property that all closed-loop systems

$$
\dot{x}=f_{i}(x)+p(x) g_{i}(x), \quad i \in \mathcal{I}=\{1,2, \ldots, m\}
$$

are continuous right side on $\mathbb{R}^{n}$ and have the origin globally asymptotically stable.

For each $i \in\{1,2, \ldots, m\}$, let $V_{i}$ be a $C^{1}$ function defined on $\mathbb{R}^{n}$. Its derivative, denoted by $\dot{V}_{i}$, along the trajectories of system $S_{i}$ can be written as

$$
\dot{V}_{i}(x)=a_{i}(x)+b_{i}(x) u,
$$

where we use from now on the notations

$$
\begin{aligned}
& a_{i}(x)=\left\langle f_{i}(x), \nabla V_{i}(x)\right\rangle, \\
& b_{i}(x)=\left\langle g_{i}(x), \nabla V_{i}(x)\right\rangle .
\end{aligned}
$$

Definition 2.1. ([6]). For each $i \in\{1,2, \ldots, m\}$, a positive definite and proper function $V_{i} \in C^{1}\left(\mathbb{R}^{n}, \mathbb{R}^{+}\right)$is said:

1) to be a control Lyapunov function (CLF) for the system $S_{i}$, with controls in $\mathcal{B}_{1}$, if

$$
\inf _{u \in \mathcal{B}_{1}} \dot{V}_{i}(x)=\inf _{u \in \mathcal{B}_{1}}\left(a_{i}(x)+b_{i}(x) u\right)<0, \quad \forall x \in \mathbb{R}^{n} \backslash\{0\} .
$$

2) to satisfy the small control property $(S C P)$ with system $S_{i}$, if for each $\varepsilon>0$ there is $\delta>0$ such that, if $x \neq 0$ satisfies $\|x\|<\delta$, then there is some $u$ with $|u|<\varepsilon$ such that

$$
\dot{V}_{i}(x)=a_{i}(x)+b_{i}(x) u<0 .
$$

Notice that $V$ is proper means that $V(x) \rightarrow \infty$ as $\|x\| \rightarrow \infty$. 
It has been shown by Lin and Sontag [6], that if $V_{i}(x)$ is a CLF for system $S_{i}$, $i \in\{1,2, \ldots, m\}$, with controls in $\mathcal{B}_{1}$, that satisfies the small control property, then the feedback

$$
u_{i}(x)= \begin{cases}-\frac{a_{i}(x)+\sqrt{a_{i}^{2}(x)+b_{i}^{4}(x)}}{b_{i}(x)\left(1+\sqrt{1+b_{i}^{2}(x)}\right)}, & \text { if } b_{i}(x) \neq 0, \\ 0, & \text { if } b_{i}(x)=0 .\end{cases}
$$

is continuous on $\mathbb{R}^{n}$ and globally asymptotically stabilizes system $S_{i}$, and it takes values in $\mathcal{B}_{1}$.

\section{Simultaneous stabilization with bounded con- trols}

This section investigates simultaneous stabilization problem. In other words, we want to find a continuous function $k($.$) such that the state feedback controller$ $u=k(x)$ globally asymptotically stabilizes the collection of systems $S_{i}$ in (1) simultaneously.

We introduce for consideration the following sets and functions. Suppose that $V_{i}$ is a CLF for system $S_{i}, i \in\{1,2, \ldots, m\}$, with controls in $\mathcal{B}_{1}$. Let

$$
\begin{array}{ll}
\mathcal{I}_{p}(x)=\{i \in\{1,2, \ldots, m\}, & \left.b_{i}(x)>0\right\} \\
\mathcal{I}_{n}(x)=\{i \in\{1,2, \ldots, m\}, & \left.b_{i}(x)<0\right\} .
\end{array}
$$

For each $i \in\{1,2, \ldots, m\}$, define $\varphi_{1 i}(x), \varphi_{2 i}(x), \psi_{1 i}(x)$ and $\psi_{2 i}(x)$ as follows

$$
\begin{gathered}
\varphi_{1 i}(x)= \begin{cases}-\frac{a_{i}(x)}{b_{i}(x)} & \text { if } \quad b_{i}(x)<0, \\
-\infty & \text { if } \quad b_{i}(x) \geq 0,\end{cases} \\
\varphi_{2 i}(x)= \begin{cases}-\frac{a_{i}(x)}{b_{i}(x)} & \text { if } b_{i}(x)>0, \\
+\infty & \text { if } b_{i}(x) \leq 0,\end{cases}
\end{gathered}
$$

and

$$
\psi_{1 i}(x)= \begin{cases}u_{i}(x) & \text { if } \quad b_{i}(x)<0 \\ 0 & \text { if } \quad b_{i}(x) \geq 0\end{cases}
$$




$$
\psi_{2 i}(x)= \begin{cases}u_{i}(x) & \text { if } \quad b_{i}(x)>0 \\ 0 & \text { if } \quad b_{i}(x) \leq 0\end{cases}
$$

Now, let

$$
\varphi_{1}(x)=\max _{i \in\{1,2, \ldots, m\}} \varphi_{1 i}(x), \quad \varphi_{2}(x)=\min _{i \in\{1,2, \ldots, m\}} \varphi_{2 i}(x)
$$

and

$$
\psi_{1}(x)=\max _{i \in\{1,2, \ldots, m\}} \psi_{1 i}(x), \quad \psi_{2}(x)=\min _{i \in\{1,2, \ldots, m\}} \psi_{2 i}(x) .
$$

Finally, let

$$
w_{1}(x)=\min \left(\psi_{1}(x), \varphi_{2}(x)\right)
$$

and

$$
w_{2}(x)=\max \left(\psi_{2}(x), \varphi_{1}(x)\right) .
$$

Remark 3.1. Since $a_{i}(x)+\sqrt{a_{i}^{2}(x)+b_{i}^{4}(x)}>0$ it follows that $b_{i}(x) u_{i}(x)<0$, if $b_{i}(x) \neq 0$. So, taking into account that $-1<u_{i}(x)<1$, one has

$$
0<u_{i}(x)<1 \text {, if } b_{i}(x)<0 \text {, and then } 0 \leq \psi_{1}(x)<1 \text {, }
$$

and

$$
-1<u_{i}(x)<0, \text { if } b_{i}(x)>0, \quad \text { and then } \quad-1<\psi_{2}(x) \leq 0 \text {. }
$$

Now, we give our necessary and sufficient stabilizability condition.

Assumption 3.1. For every $x \in \mathbb{R}^{n} \backslash\{0\}, \varphi_{1}(x)<\varphi_{2}(x)$.

Remark 3.2. Note that Assumption 3.1: for each $x \in \mathbb{R}^{n}, \varphi_{1}(x)<\varphi_{2}(x)$ is equivalent to

$$
\begin{aligned}
& \max _{i \in \mathcal{I}_{n}(x)}\left(-\frac{a_{i}(x)}{b_{i}(x)}\right)<\min _{i \in \mathcal{I}_{p}(x)}\left(-\frac{a_{i}(x)}{b_{i}(x)}\right), \\
& \forall x \in \mathbb{R}^{n}, \text { s. t. } \mathcal{I}_{n}(x) \neq \emptyset \text { and } \mathcal{I}_{p}(x) \neq \emptyset .
\end{aligned}
$$

If for every $i \in\{1,2, \ldots, m\}$, system $S_{i}$ has a CLF with controls not necessary in $\mathcal{B}_{1}$, that is

$$
\inf _{u \in \mathbb{R}} \dot{V}_{i}(x)=\inf _{u \in \mathbb{R}}\left(a_{i}(x)+b_{i}(x) u\right)<0, \quad \forall x \in \mathbb{R}^{n} \backslash\{0\},
$$

and based on Sontag's formula [14], Wu in [18] constructed an unbounbed simultaneous stabilizing controller for systems in (1) if Assumption 3.1 holds. Later, under the assumption that for each $x \in \mathbb{R}^{n}$, all the functions $b_{i}(x)$ have the same 
sign, Zhang et al. [21] proposed another unbounbed simultaneously stabilizing feedback for systems in (1).

Next, we give the main result of this paper.

Theorem 3.1. There exists a continuous feedback $k: \mathbb{R}^{n} \rightarrow \mathcal{B}_{1}$ that globally asymptotically stabilizes simultaneously the collection of systems in (1), if and only if, there exist a collection of control Lyapunov functions $V_{i}, i=1,2, \ldots, m$, with controls in $\mathcal{B}_{1}$, satisfying the small control property, such that Assumption 3.1 holds.

Moreover, the feedback

$$
u(x)=\frac{w_{1}(x)+w_{2}(x)}{2}
$$

is continuous, takes values in $\mathcal{B}_{1}$ and globally asymptotically stabilizes simultaneously the collection of systems in (1).

Proof. Necessity: Similarly to the proof of Theorem 1 in [18], we can show that if there exists a simultaneously continuous stabilizing feedback $k: \mathbb{R}^{n} \rightarrow \mathcal{B}_{1}$ for the collection of systems in (1), then, for every $i \in\{1,2, \ldots, m\}$, there exists a CLF $V_{i}$ for system $S_{i}$ with controls in $\mathcal{B}_{1}$ and which satisfies the small control property. Moreover, Assumption 3.1, $\varphi_{1}(x)<\varphi_{2}(x), \quad \forall x \in \mathbb{R}^{n}$, holds.

Sufficiency: We first prove the continuity of $w_{1}$. From the continuity of functions $u_{i}$ and, since $u_{i}(x)=0$ whenever $b_{i}(x)=0$ for all $i=1,2, \ldots, m$, we deduce that $\psi_{1 i}$ is continuous for all $i=1,2, \ldots, m$, and consequently, $\psi_{1}$ is continuous on $\mathbb{R}^{n}$.

Let $x_{0} \in \mathbb{R}^{n} \backslash\{0\}$. For each $i \in\{1,2, \ldots, m\}$, we will study the continuity of $\varphi_{2 i}$ at $x_{0}$ according to the sign of $b_{i}\left(x_{0}\right)$.

- If $b_{i}\left(x_{0}\right)=0$, then $a_{i}\left(x_{0}\right)<0$ since $V_{i}$ is a CLF for the system $S_{i}$. By continuity of the function $a_{i}, a_{i}(x)<0$ in a neighborhood $\mathcal{V}_{x_{0}}$ of $x_{0}$.

For $x \in \mathcal{V}_{x_{0}}$ such that $b_{i}(x)>0$, we have $0<-\frac{a_{i}(x)}{b_{i}(x)}=\varphi_{2 i}(x)$ and then

$$
\lim _{\substack{x \rightarrow x_{0} \\ b_{i}(x)>0}} \varphi_{2 i}(x)=\lim _{\substack{x \rightarrow x_{0} \\ b_{i}(x)>0}}-\frac{a_{i}(x)}{b_{i}(x)}=+\infty .
$$

For $x \in \mathcal{V}_{x_{0}}$ such that $b_{i}(x)<0$, we have $\varphi_{2 i}(x)=+\infty$.

Therefore in both cases, the following holds

$$
\forall L>0, \quad \exists \lambda_{L}^{i}>0 \text { s. t. } \varphi_{2 i}(x)>L, \quad \forall x \in B\left(x_{0}, \lambda_{L}^{i}\right) .
$$


- If $b_{i}\left(x_{0}\right)<0$, then by continuity $b_{i}(x)<0$ in a neighborhood $\mathcal{W}_{x_{0}}$ of $x_{0}$. Therefore, $\varphi_{2 i}(x)=+\infty$ on $\mathcal{W}_{x_{0}}$ and then (7) remains valid in the case $b_{i}\left(x_{0}\right)<0$.

Hence the assertion ( 7$)$ holds for any $i \notin \mathcal{I}_{p}\left(x_{0}\right)$, i.e., for any $i \in\{1,2, \ldots, m\}$ such that $b_{i}\left(x_{0}\right) \leq 0$.

Choosing $\lambda_{L}=\min _{i \notin \mathcal{I}_{p}\left(x_{0}\right)} \lambda_{L}^{i}$, it follows for all $i \notin \mathcal{I}_{p}\left(x_{0}\right)$

$$
\forall L>0, \quad \exists \lambda_{L}>0 \text { s. t. } \varphi_{2 i}(x)>L, \quad \forall x \in B\left(x_{0}, \lambda_{L}\right) .
$$

- If $b_{i}\left(x_{0}\right)>0$, then the continuity of the map $b_{i}$ ensures the existence of $\delta_{i}>0$ such that $b_{i}(x)>\frac{b_{i}\left(x_{0}\right)}{2}>0$ on $B\left(x_{0}, \delta_{i}\right)$. It follows that $\varphi_{2 i}(x)=-\frac{a_{i}(x)}{b_{i}(x)}$ is continuous and bounded on $B\left(x_{0}, \delta_{i}\right)$. By choosing $\delta=\min _{i \in \mathcal{I}_{p}\left(x_{0}\right)} \delta_{i}$, one has, for any $i \in \mathcal{I}_{p}\left(x_{0}\right), \varphi_{2 i}$ is continuous on $B\left(x_{0}, \delta\right)$ and for all $x \in B\left(x_{0}, \delta\right)$

$$
\varphi_{2 i}(x)=-\frac{a_{i}(x)}{b_{i}(x)} \leq L_{1}=\max _{j \in \mathcal{I}_{p}\left(x_{0}\right)}\left(\sup _{y \in B\left(x_{0}, \delta\right)} \varphi_{2 j}(y)\right) .
$$

Now, if $\mathcal{I}_{p}\left(x_{0}\right)=\emptyset$, that is, $b_{i}\left(x_{0}\right) \leq 0$ for all $i=1,2, \ldots, m$, then from (8), together with the fact that $\psi_{1}$ is continuous and positive, it follows that for any $\varepsilon>0$, one can choose $0<\lambda_{L_{0}}<\varepsilon$, such that for all $i \in\{1,2, \ldots, m\}$

$$
\varphi_{2 i}(x)>L_{0}=\sup _{y \in B\left(x_{0}, \varepsilon\right)} \psi_{1}(y), \quad \forall x \in B\left(x_{0}, \lambda_{L_{0}}\right) .
$$

Therefore, for all $x \in B\left(x_{0}, \lambda_{L_{0}}\right)$ we have

$$
\varphi_{2}(x)=\min _{i \in\{1,2, \ldots, m\}} \varphi_{2 i}(x)>L_{0} \geq \psi_{1}(x),
$$

which clearly implies that $w_{1}(x)=\psi_{1}(x)$, for all $x \in B\left(x_{0}, \lambda_{L_{0}}\right)$. Thus $w_{1}$ is continuous at $x_{0}$.

If $\mathcal{I}_{p}\left(x_{0}\right) \neq \emptyset$, then by using (8) and (9), and taking $\eta=\min \left(\lambda_{L_{1}}, \delta\right)$, one gets

$$
\begin{aligned}
& \varphi_{2 i}(x) \leq L_{1}<\varphi_{2 j}(x), \\
& \forall x \in B\left(x_{0}, \eta\right), \quad \forall i \in \mathcal{I}_{p}\left(x_{0}\right), \text { and } \forall j \notin \mathcal{I}_{p}\left(x_{0}\right) .
\end{aligned}
$$

It then follows that for all $x \in B\left(x_{0}, \eta\right)$

$$
\varphi_{2}(x)=\min _{i \in\{1,2, \ldots, m\}} \varphi_{2 i}(x)=\min _{i \in \mathcal{I}_{p}\left(x_{0}\right)} \varphi_{2 i}(x)
$$

and consequently $\varphi_{2}$ is continuous on $B\left(x_{0}, \eta\right)$. From this, for all $x \in B\left(x_{0}, \eta\right)$, $w_{1}(x)=\min \left(\psi_{1}(x), \varphi_{2}(x)\right)$. Thus, $w_{1}$ is continuous at $x_{0}$. 
Finally, assume that $V_{i}, i \in\{1,2, \ldots, m\}$, satisfies the small control property. We wish to show that the function $w_{1}$ is continuous at the origin.

For each $i \in\{1,2, \ldots, m\}$, from the definition of $\varphi_{2 i}$ and the small control property of $V_{i}$, it is not difficult to obtain that

$$
\forall \varepsilon>0, \quad \exists \delta_{i}>0 \text { such that, }-\varepsilon<\varphi_{2 i}(x), \quad \forall x \in B\left(0, \delta_{i}\right) .
$$

Take $\delta=\min _{i \in\{1,2, \ldots, m\}} \delta_{i}$, it follows

$$
\forall \varepsilon>0, \quad \exists \delta>0 \text { such that, }-\varepsilon<\varphi_{2}(x), \quad \forall x \in B(0, \delta) .
$$

Continuity of $\psi_{1}$ at the origin leads to

$$
\forall \varepsilon>0, \quad \exists \gamma>0 \text { such that, } \quad-\varepsilon<\psi_{1}(x)<\varepsilon, \quad \forall x \in B(0, \gamma) .
$$

Take $\eta=\min (\delta, \gamma)$, it follows from (10) and (11)

$$
\begin{aligned}
& \forall \varepsilon>0, \quad \exists \eta>0 \quad \text { such that, } \\
& -\varepsilon<w_{1}(x)=\min \left(\psi_{1}(x), \varphi_{2}(x)\right)<\varepsilon, \quad \forall x \in B(0, \eta) .
\end{aligned}
$$

That is, $w_{1}$ is continuous at the origin and so on $\mathbb{R}^{n}$.

The continuity of $w_{2}$ on $\mathbb{R}^{n}$ can be treated similarly and is omitted here.

For the global asymptotic stability, we only have to verify that for all $i=$ $1,2, \ldots, m$,

$$
\dot{V}_{i}(x)=a_{i}(x)+b_{i}(x) u(x)<0, \quad \forall x \in \mathbb{R}^{n} \backslash\{0\},
$$

according to the sign of $b_{i}(x)$.

- If $b_{i}(x)>0$. On the one hand, one has $w_{1}(x)=\min \left(\psi_{1}(x), \varphi_{2}(x)\right) \leq \varphi_{2}(x) \leq$ $-\frac{a_{i}(x)}{b_{i}(x)}$. On the other hand, one has, $\psi_{2}(x) \leq u_{i}(x)<-\frac{a_{i}(x)}{b_{i}(x)}$ and, from Assumption 3.1 , one has $\varphi_{1}(x)<\varphi_{2}(x) \leq-\frac{a_{i}(x)}{b_{i}(x)}$. Hence,

$$
w_{2}(x)=\max \left(\psi_{2}(x), \varphi_{1}(x)\right)<-\frac{a_{i}(x)}{b_{i}(x)} .
$$

The conclusion follows since $u(x)=\frac{w_{1}(x)+w_{2}(x)}{2}<-\frac{a_{i}(x)}{b_{i}(x)}$.

- If $b_{i}(x)<0$. Similarly to the previous case, on one hand, one has $w_{2}(x)=$ $\max \left(\psi_{2}(x), \varphi_{1}(x)\right) \geq \varphi_{1}(x) \geq-\frac{a_{i}(x)}{b_{i}(x)}$.

On the other hand, one has, $\psi_{1}(x) \geq u_{i}(x)>-\frac{a_{i}(x)}{b_{i}(x)}$ and, from Assumption 3.1, one has $\varphi_{2}(x)>\varphi_{1}(x) \geq-\frac{a_{i}(x)}{b_{i}(x)}$. Again, the conclusion follows since $w_{1}(x)=\min \left(\psi_{1}(x), \varphi_{2}(x)\right)>-\frac{a_{i}(x)}{b_{i}(x)}$. 
- If $b_{i}(x)=0$, then $\dot{V}_{i}(x)=a_{i}(x)<0$, since $V_{i}(x)$ is a CLF for the system $S_{i}$.

Thus, $\dot{V}_{i}(x)$ is negative definite as desired.

Now, let $x$ in $\mathbb{R}^{n}$, we shall verify that $-1<u(x)<1$. To this end we only have to verify $-1<w_{1}(x)<1$ and $-1<w_{2}(x)<1$.

To verify $-1<w_{1}(x)<1$, we distinguish the cases $\mathcal{I}_{p}(x)=\emptyset$ and $\mathcal{I}_{p}(x) \neq \emptyset$.

- If $\mathcal{I}_{p}(x)=\emptyset$, then $b_{i}(x) \leq 0$ for all $i=1,2, \ldots, m$. Therefore, $\varphi_{2 i}(x)=+\infty$ for all $i=1,2, \ldots, m$. Consequently, $\varphi_{2}(x)=+\infty$. From this and since $0 \leq$ $\psi_{1}(x)<1$ (see Remark 3.1), we get

$$
w_{1}(x)=\min \left(\psi_{1}(x), \varphi_{2}(x)\right)=\psi_{1}(x) .
$$

So, $0 \leq w_{1}(x)<1$.

- If $\mathcal{I}_{p}(x) \neq \emptyset$, then $b_{i}(x)>0$ for all $i \in \mathcal{I}_{p}(x)$. Notably, $\varphi_{2 i}(x)=-\frac{a_{i}(x)}{b_{i}(x)}$ is finite for all $i \in \mathcal{I}_{p}(x)$. So, let $i_{0} \in \mathcal{I}_{p}(x)$ is such that $-\frac{a_{i_{0}}(x)}{b_{i_{0}}(x)}=\min _{i \in \mathcal{I}_{p}(x)}\left(-\frac{a_{i}(x)}{b_{i}(x)}\right)$, that is, $\varphi_{2 i_{0}}(x)=\min _{i \in \mathcal{I}_{p}(x)} \varphi_{2 i}(x)$. But, $\varphi_{2 i}(x)=+\infty$ if $b_{i}(x) \leq 0$, i.e. for $i \notin \mathcal{I}_{p}(x)$, it then follows

$$
\varphi_{2}(x)=\min _{i \in\{1,2, \ldots, m\}} \varphi_{2 i}(x)=\min _{i \in \mathcal{I}_{p}(x)} \varphi_{2 i}(x)=-\frac{a_{i_{0}}(x)}{b_{i_{0}}(x)} .
$$

Recall that the feedback $u_{i_{0}}$, defined by (4), stabilizes the system $S_{i_{0}}$ and takes values in $\mathcal{B}_{1}$, consequently, $a_{i_{0}}(x)+b_{i_{0}}(x) u_{i_{0}}(x)<0$, so $u_{i_{0}}(x)<-\frac{a_{i_{0}}(x)}{b_{i_{0}}(x)}$ since $b_{i_{0}}(x)>0$. Hence

$$
-1<u_{i_{0}}(x)<-\frac{a_{i_{0}}(x)}{b_{i_{0}}(x)}=\varphi_{2}(x) .
$$

This last inequality and the fact that $0 \leq \psi_{1}(x)<1$ (see Remark 3.1) lead to

$$
-1<\min \left(\psi_{1}(x), \varphi_{2}(x)\right)=w_{1}(x)<1 .
$$

In both cases $-1<w_{1}(x)<1$, for all $x \in \mathbb{R}^{n}$.

Now, we still have to verify $-1<w_{2}(x)<1$. As above, we distinguish the cases $\mathcal{I}_{n}(x)=\emptyset$ and $\mathcal{I}_{n}(x) \neq \emptyset$. 
- If $\mathcal{I}_{n}(x)=\emptyset$, then $b_{i}(x) \geq 0$ for all $i=1,2, \ldots, m$. Therefore, $\varphi_{1 i}(x)=-\infty$ for all $i=1,2, \ldots, m$. Consequently, $\varphi_{1}(x)=-\infty$. From this and since $-1<$ $\psi_{2}(x) \leq 0$ (see Remark 3.1), we get

$$
w_{2}(x)=\max \left(\psi_{2}(x), \varphi_{1}(x)\right)=\psi_{2}(x) .
$$

So, $-1<w_{2}(x) \leq 0$.

- If $\mathcal{I}_{n}(x) \neq \emptyset$, then $b_{i}(x)<0$ for all $i \in \mathcal{I}_{n}(x)$. Let $i_{0} \in \mathcal{I}_{n}(x)$ be such that $-\frac{a_{i_{0}}(x)}{b_{i_{0}}(x)}=\max _{i \in \mathcal{I}_{n}(x)}\left(-\frac{a_{i}(x)}{b_{i}(x)}\right)$. It then follows

$$
\varphi_{1}(x)=\max _{i \in\{1,2, \ldots, m\}} \varphi_{1 i}(x)=\max _{i \in \mathcal{I}_{n}(x)} \varphi_{1 i}(x)=-\frac{a_{i_{0}}(x)}{b_{i_{0}}(x)}
$$

since $\varphi_{1 i}(x)=-\infty$ if $b_{i}(x) \geq 0$, i.e. $i \notin \mathcal{I}_{n}(x)$.

Recall that the feedback $u_{i_{0}}$, defined by (4), stabilizes the system $S_{i_{0}}$ and takes values in $\mathcal{B}_{1}$, consequently, $a_{i_{0}}(x)+b_{i_{0}}(x) u_{i_{0}}(x)<0$, so $u_{i_{0}}(x)>-\frac{a_{i_{0}}(x)}{b_{i_{0}}(x)}$ since $b_{i_{0}}(x)<0$. This implies $\varphi_{1}(x)=-\frac{a_{i_{0}}(x)}{b_{i_{0}}(x)}<u_{i_{0}}(x)<1$. On the other hand, we have $-1<\psi_{2}(x) \leq 0$ (see Remark 3.1). We then obtain

$$
-1<w_{2}(x)=\max \left(\psi_{2}(x), \varphi_{1}(x)\right)<1 .
$$

In both cases $-1<w_{2}(x)<1$, for all $x \in \mathbb{R}^{n}$.

We have then proved that the stabilizing feedback $u$ defined by (5) takes values in $\mathcal{B}_{1}$ and this completes the proof of Theorem 3.1.

\section{Example}

Consider a system with the following two possible modes

$$
\begin{gathered}
S_{1}:\left\{\begin{array}{l}
\dot{x}_{1}=x_{2}^{3}+\frac{-2 x_{1}^{2}-2 x_{2}^{2}}{1+x_{1}^{2}}+u\left(-2 x_{1}+x_{2}\right) \\
\dot{x}_{2}=-x_{2}^{3}+\frac{x_{1}^{2}+x_{2}^{2}}{1+x_{1}^{2}}+u\left(x_{1}-x_{2}\right),
\end{array}\right. \\
S_{2}:\left\{\begin{array}{l}
\dot{x}_{1}=\frac{-x_{1}^{3}-x_{1} x_{2}^{2}+x_{1}^{2}}{1+x_{1}^{2}}+u x_{1} \\
\dot{x}_{2}=\frac{x_{1} x_{2}}{1+x_{1}^{2}}+u x_{2} .
\end{array}\right.
\end{gathered}
$$


We want to find a continuous function $p: \mathbb{R}^{2} \rightarrow \mathcal{B}_{1}$ such that the state feedback controller $u(x)=p(x)$ globally asymptotically stabilizes the two systems (13) and (14).

Let

$$
V_{1}(x)=\frac{\left(x_{1}+x_{2}\right)^{2}}{2}+\frac{x_{2}^{2}}{2},
$$

and

$$
V_{2}(x)=\frac{x_{1}^{2}+x_{2}^{2}}{2}
$$

The Lie derivative of $V_{1}$ and $V_{2}$ along the trajectories of systems $S_{1}$ and $S_{2}$, respectively, write:

$$
\dot{V}_{1}(x)=a_{1}(x)+b_{1}(x) u, \text { and } \dot{V}_{2}(x)=a_{2}(x)+b_{2}(x) u,
$$

with

$$
a_{1}(x)=-x_{2}^{4}+x_{1}\left(\frac{-x_{1}^{2}-x_{2}^{2}}{1+x_{1}^{2}}\right), \quad b_{1}(x)=-x_{1}^{2}-x_{2}^{2},
$$

and

$$
a_{2}(x)=-x_{1}^{2}\left(\frac{x_{1}^{2}+x_{2}^{2}}{1+x_{1}^{2}}\right)+x_{1}\left(\frac{x_{1}^{2}+x_{2}^{2}}{1+x_{1}^{2}}\right), \quad b_{2}(x)=x_{1}^{2}+x_{2}^{2} .
$$

It can be seen that $V_{1}$ is a CLF for sysytem (13) and that $V_{2}$ is a CLF for sysytem (14). It is easy to verify that both $V_{1}$ and $V_{2}$ satisfy the the small control property $(\mathrm{SCP})$. On the other hand, straightforward calculations lead to

$$
\frac{a_{1}(x)}{b_{1}(x)}=\frac{x_{2}^{4}}{x_{1}^{2}+x_{2}^{2}}+\frac{x_{1}}{1+x_{1}^{2}} \text { and } \frac{a_{2}(x)}{b_{2}(x)}=\frac{-x_{1}^{2}+x_{1}}{1+x_{1}^{2}} .
$$

Observe that $-\frac{a_{1}(x)}{b_{1}(x)}<1$ and $-1<-\frac{a_{2}(x)}{b_{2}(x)}$, for all $x \in \mathbb{R}^{2} \backslash\{0\}$, and, since $b_{1}(x)<0$, $b_{2}(x)>0, x \neq 0$, it follows

$$
\inf _{u \in \mathcal{B}_{1}} \dot{V}_{1}(x)<0, \text { and } \inf _{u \in \mathcal{B}_{1}} \dot{V}_{2}(x)<0, \quad \forall x \in \mathbb{R}^{2} \backslash\{0\} .
$$

We can verify that

$$
\frac{a_{2}(x)}{b_{2}(x)}-\frac{a_{1}(x)}{b_{1}(x)}=-\frac{x_{1}^{2}}{1+x_{1}^{2}}-\frac{x_{2}^{4}}{x_{1}^{2}+x_{2}^{2}}<0, \quad \forall x \in \mathbb{R}^{2} \backslash\{0\},
$$

that is, Assumption 3.1, $\varphi_{1}(x)<\varphi_{2}(x)$, for all $x \in \mathbb{R}^{2}$, is satisfied. Therefore, according to Theorem 3.1, the feedback

$$
u(x)=\frac{w_{1}(x)+w_{2}(x)}{2},
$$


with

$$
w_{1}(x)= \begin{cases}\min \left(-\frac{a_{1}(x)+\sqrt{a_{1}^{2}(x)+b_{1}^{4}(x)}}{b_{1}(x)\left(1+\sqrt{1+b_{1}^{2}(x)}\right)},-\frac{a_{2}(x)}{b_{2}(x)}\right) & \text { if } x \neq 0, \\ 0, & \text { if } x=0\end{cases}
$$

and

$$
w_{2}(x)= \begin{cases}\max \left(-\frac{a_{2}(x)+\sqrt{a_{2}^{2}(x)+b_{2}^{4}(x)}}{b_{2}(x)\left(1+\sqrt{1+b_{2}^{2}(x)}\right)},-\frac{a_{1}(x)}{b_{1}(x)}\right) & \text { if } x \neq 0, \\ 0, & \text { if } x=0\end{cases}
$$

$x$

is continuous on $\mathbb{R}^{2}$, takes values in $\mathcal{B}_{1}$ and globally asymptotically stabilizes systems $S_{1}$ and $S_{2}$.

The behaviors of the closed-loop systems (13) and (14) under the action of the simultaneously bounded stabilizing feedback are drawn in Figures 1, 3 and Figure 5. The evolution of the stabilizing feedback along the closed-loop systems (13) and (14) is shown in Figure 2 and Figure 4.

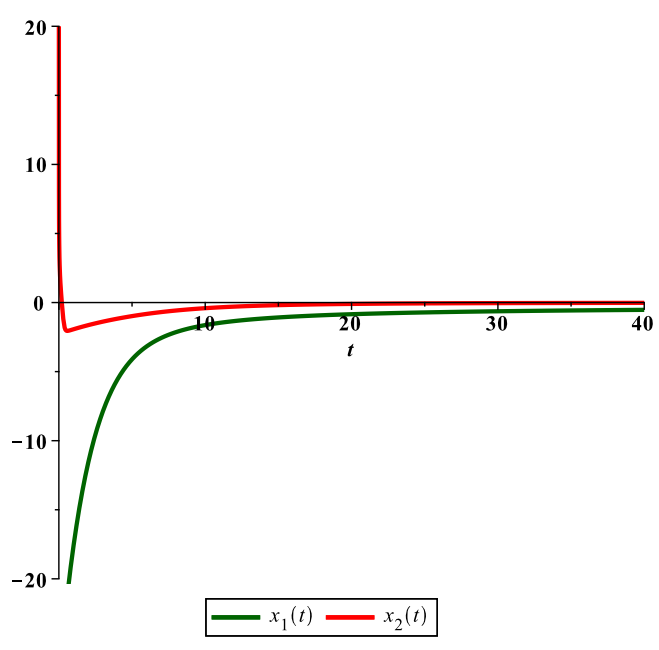

Figure 1: Evolution of solutions of system (13) with the feedback given by the formula (15). 


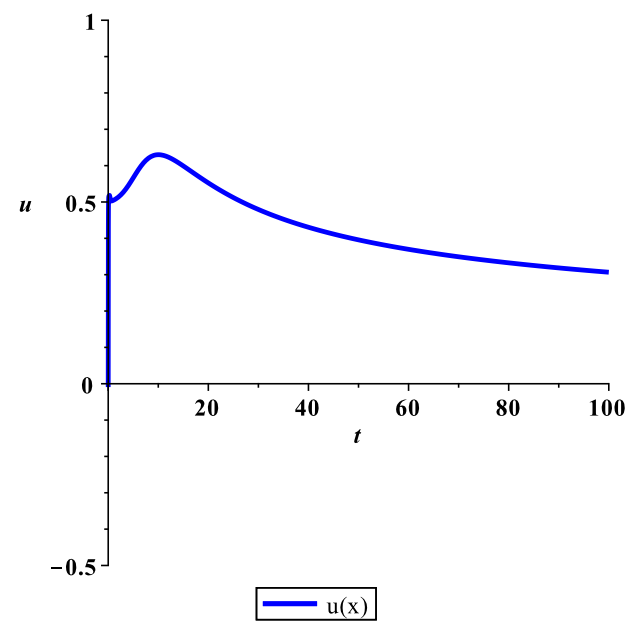

Figure 2: Evolution of the stabilizing feedback $u\left(x_{1}, x_{2}\right)$ applied to system (13).

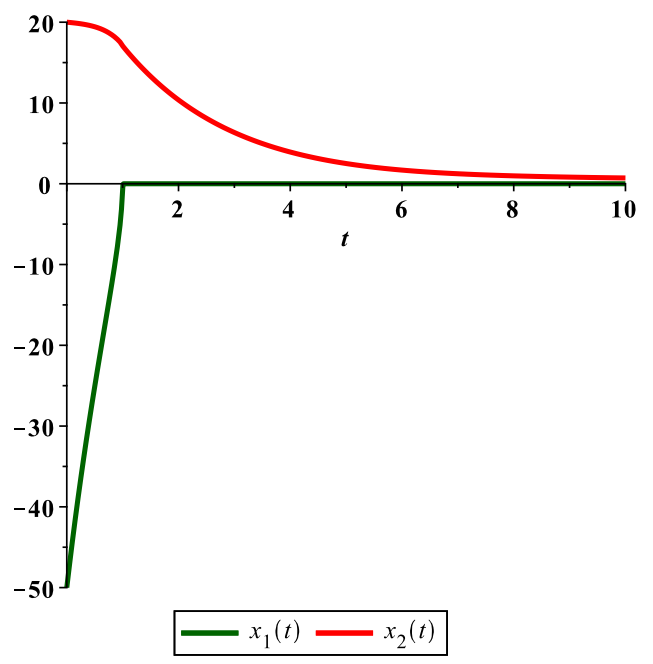

Figure 3: Evolution of solutions of System (14) with the feedback given by the formula (15). 


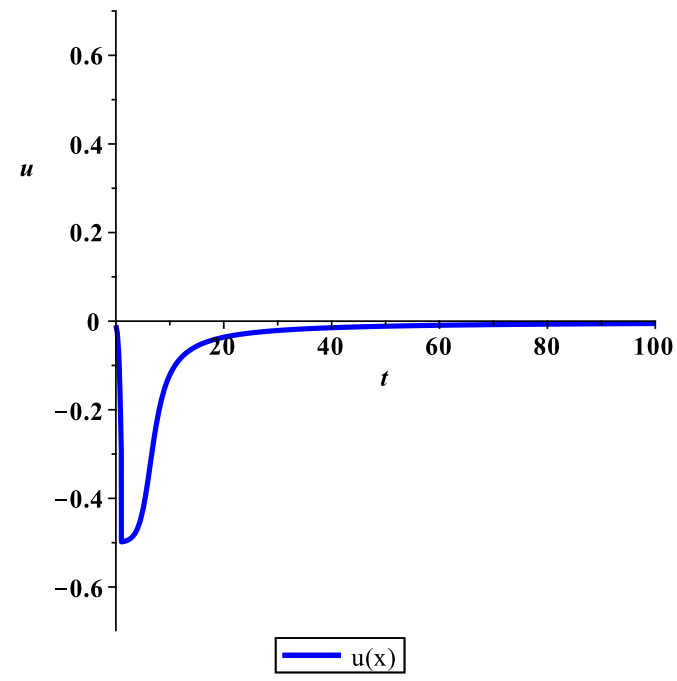

Figure 4: Evolution of the stabilizing feedback $u\left(x_{1}, x_{2}\right)$ applied to System (14).

\section{Conclusion}

In this paper, we have studied the problem of simultaneous stabilization of nonlinear systems with bounded controls. Under the assumption that a collection of control Lyapunov functions (CLFs), with controls in the unit interval, are known, we gave a necessary and sufficient condition for the simultaneous stabilization. Moreover, an explicit formula for constructing simultaneously bounded stabilizing feedback laws was proposed.

\section{References}

[1] Z. Artstein, "Stabilization with relaxed controls", Nonlinear Analysis Theory Methods and applications, vol. 7, pp. 1163-1173, 1983.

[2] Y. Y. Cao and J. Lam, "A computational methods for simultaneous LQ optimal control design via piecewise constant output feedback", IEEE Trans. Syst., Man, Cybern. B: Cybern., vol. 31, pp. 836-842, 2001. 


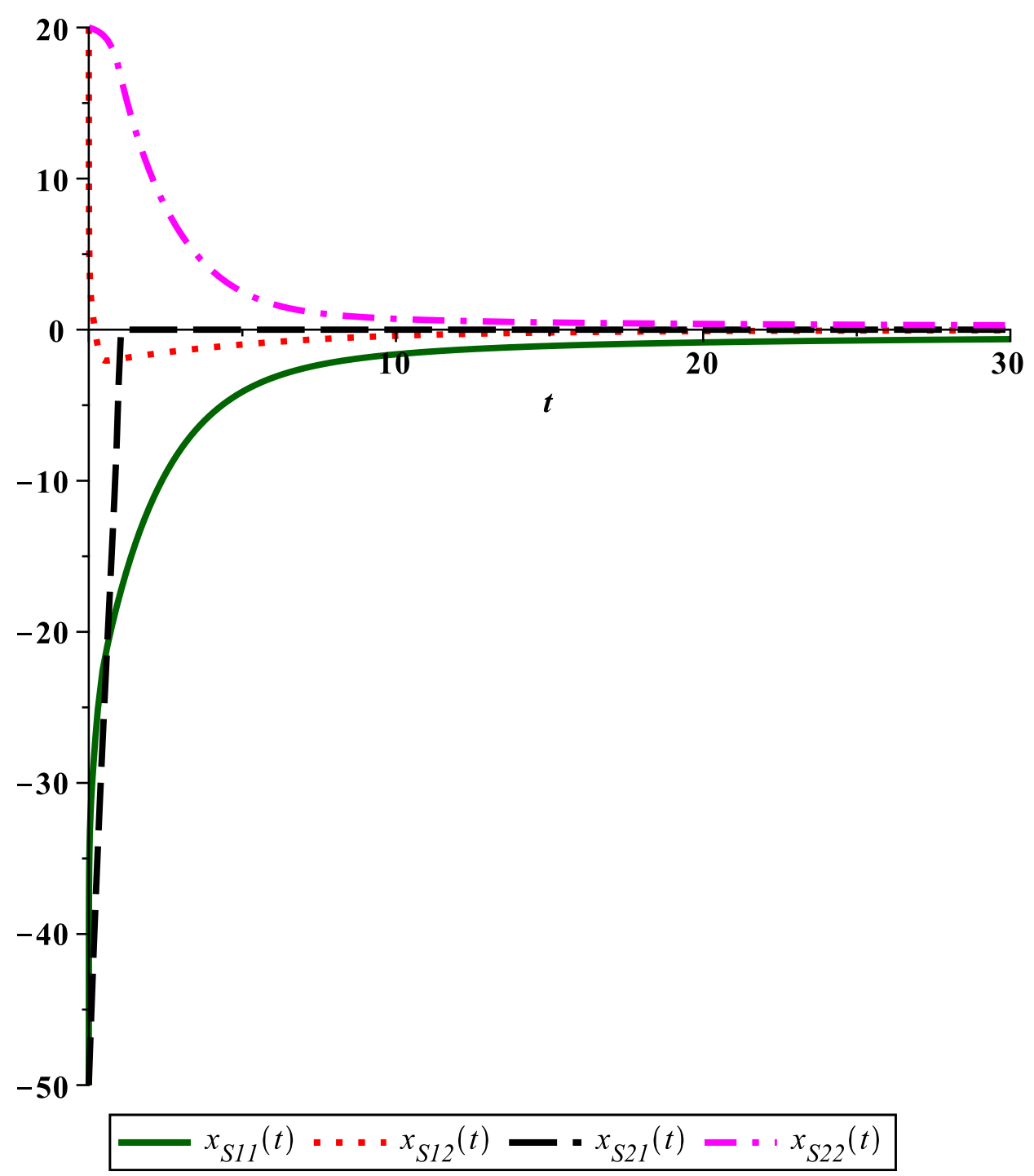

Figure 5: Evolution of solutions of System (13) and System (14) under the action of the simultaneously bounded stabilizing feedback. 
[3] Y. Y. Cao, Y. X. Sun, and J. Lam, "Simultaneous stabilization via static output feedback and state feedback," IEEE Trans. Autom. Control, vol. 44, pp. 1277-1282, 1999.

[4] B, Ho-Mock-Qai and W. P. Dayawansa, "Simultaneous stabilization of linear and nonlinear systems by means of nonlinear state feedback", SIAM J Control Optim, vol. 37, pp. 1701-1725, 1999.

[5] P. T. Kabamba and C. Yang, (1991). "Simultaneous controller design for linear time-invariant systems", IEEE Trans. Autom. Control, vol. 36, pp. 106-110, 1991.

[6] Y. Lin and E. D. Sontag, "A universal formula for stabilization with bounded controls", Systems $\&$ control letters, vol. 16, pp. 393-397, 1991.

[7] L. Maniar, M. Oumoun and J. C. Vivalda, "On the stabilization of quadratic nonlinear systems", European Journal of Control, vol. 35, pp. 28-33, 2017.

[8] D. E. Miller and T. Chen, "Simultaneous stabilization with near-optimal $H_{\infty}$ performance", IEEE Trans. Autom. Control, vol. 47, pp. 1986-1998, 2002.

[9] D. E. Miller and M. Rossi, "Simultaneous stabilization with near-optimal LQR performance", IEEE Trans. Autom. Control, vol. 46, pp. 1543-1555, 2001.

[10] E. Moulay, W. Perruquetti, "Stabilization of nonaffine systems: A constructive method for polynomial systems", IEEE Transactions on Automatic Control, vol. 50, pp. 520-526, 2005.

[11] L. Rifford, "Semiconcave control-Lyapunov functions and stabilizing feedbacks," SIAM J. Control Optim., vol. 41, pp. 659-681, 2002.

[12] R. Saeks and J. Murray, "Fractional representation, algebraic geometry, and the simultaneous stabilization problem", IEEE Trans. Autom. Control, vol. 27, pp. 895-903, 1982.

[13] W. E. Schmitendorf and C. C. Hollot, "Simultaneous stabilization via linear state feedback control",IEEE Trans. Autom. Control, vol. 34, pp. 1001-1005, 1989.

[14] E. D. Sontag, "A universal construction of Artstein's theorem on nonlinear stabilization", Systems \& control letters, vol. 13, pp. 117-123, 1989.

[15] J. Tsinias, "Asymptotic feedback stabilization: A sufficient condition for the existence of control lyapunov functions", Systems and Control Letters, vol. 15, pp. 441-448, 1990. 
[16] J. Tsinias, "Existence of control lyapunov functions and applications to state feedback stabilizability of nonlinear systems", SIAM Journal on Control and Optimization, vol. 29, pp. 457-473, 1991.

[17] Y. Wang, G. Feng, and D. Cheng, "Simultaneous stabilization of a set of nonlinear port-controlled Hamiltonian systems", Automatica, vol. 43, pp. 403415, 2007.

[18] J. L. Wu, "Simultaneous stabilization for a collection of single-input nonlinear systems", IEEE Trans. Autom. Control, vol. 50, pp. 328-337, 2005.

[19] J. L. Wu, "Simultaneous quadratic stabilization for discrete-time nonlinear systems", IEEE Trans. Autom. Control, vol. 55, pp. 1443-1448, 2010.

[20] J. Zhong, D. Cheng, X. Hu, "Constructive stabilization for quadratic input nonlinear systems", Automatica, vol. 44, pp. 1996-2005, 2008.

[21] W. Zhang, Z. Z. Han and X. S. Cai, "Simultaneous stabilization of a collection of single-input nonlinear systems based on CLFs", Asian Journal of Control, vol. 13, pp. 582-589, 2011. 